\title{
National lockdown poses new challenges for leprosy patients in Nepal
}

\author{
S. Nepal ${ }^{\mathrm{a}}$, R. K. Paudel ${ }^{\mathrm{a}} \&$ S. Aryal ${ }^{\mathrm{a}}$ \\ ${ }^{\mathrm{a}}$ The Leprosy Mission Nepal, Nepal
}

Submitted 15 July 2020; Accepted 20 January 2021

Sir,

In 2009, the Government of Nepal announced that leprosy had been eliminated from the country-according to the WHO measurement of less than 1 case per 10,000 head of population. ${ }^{1}$ Every year around 3,200 new leprosy cases are reported in Nepal. ${ }^{2}$ The primary health care services are available at community level, while specialized services for leprosy are available at only three hospitals: Anandaban Hospital, Green Pastures Hospital, and Lalgadh Hospital. Due to the nationwide lockdown related to COVID-19, all the community level activities carried out in leprosy endemic districts have been halted to support the government in controlling the infection. All leprosy endemic districts are occupied with issues of quarantine and isolation, which have hindered leprosy control activities. The deep-rooted stigma in society is itself a major obstacle in identifying new cases of leprosy. The threat of COVID-19 has overtaken the importance of disability due to leprosy. Many new leprosy cases are under the shadow of the COVID threat. COVID cases are increasingly found in the terai areas where there is a pre-existing threat of leprosy. Local health care workers and human resources for health are busy constructing and serving in quarantine camps as a shield against COVID.

The first case of COVID-19 in Nepal was confirmed on 23rd January 2020 when a 31-yearold student, who had returned to Kathmandu from Wuhan on January 9, tested positive for the disease. ${ }^{3}$ However, 62 days after the first confirmed case in Nepal, an urgent notice regarding travel restrictions related to COVID-19 was issued and circulated by the Department of Immigration, leading to official restrictions on mass gathering at government and community level; a nationwide lockdown was imposed from March 24th. This had a major impact on leprosy treatment. When the lockdown was announced in Nepal all the essential health service priorities were shifted to COVID-19 which led to interruption of new case detection in the leprosy endemic districts of Nepal. The treatment of leprosy was adversely affected, as all modes of transportation were suspended. The satellite clinics run each month by Anandaban Hospital in Butwal and Biratnagar were also halted, which led to further problems in the detection of new leprosy cases and the management of complications such as a reactions and ulcers.

Correspondence to: Sudip Nepal, The Leprosy Mission Nepal, Nepal (e-mail: nepalsudip25@ gmail.com) 
According to a quarterly report by the health offices of leprosy endemic districts, new case detection has decreased by around 50\%. Due to the ongoing nationwide lockdown new/hidden cases of leprosy from the community have not been diagnosed, which poses the threat of disability. Although all the health facilities are open for services, due to the risk of COVID19 , leprosy related services have been affected the most. Complicated cases of ulcer and reaction from hard to reach communities are deprived of treatment due to inadequate access to transportation. Regular skin camps and contact tracing, which are the priority of the Nepal government for early case detection, have been interrupted. The lockdown has led to distress for patients from hard to reach communities and leprosy endemic districts to receive leprosy related services from centralized hospitals like Anandaban, Lalgadh, and Green Pastures hospital. The requirement for mandatory PCR tests to enter the capital city where Anandaban hospital is located, means that leprosy patients have to pay more than double the ambulance fare in comparison to normal times. These added costs of PCR testing and extra transportation have been catastrophic to leprosy patients from marginalized and hard to reach communities.

The financial crisis provoked by the lockdown is far-reaching. Leprosy-affected patients may be neglected during relief distribution. The majority of daily workers are idle due to postponed work schedules. This has a serious impact on leprosy-affected persons, as they are being neglected and abandoned by their families, with consequent mental stress.

The delay in detection and treatment of new leprosy cases is a stumbling block to our present mission, goals, and the target of Zero leprosy cases in the future. The target of ILEP's vision is 'a world free from leprosy'. ${ }^{4}$ To achieve Zero Leprosy Transmission, Zero Leprosy Disability and Zero Leprosy Stigma and Discrimination needs continuity and functioning of the health system. It is mandatory to prioritize every disease with a similar depth of seriousness because the threat of one disease cannot be an excuse for the emerging risk of other diseases. It is high time for the Nepal Government to develop specific strategies to assist leprosy-affected persons in order to prevent future distress.

\section{References}

1 Global Leprosy Update, 2010. Weekly Epidemiological Record. World Health Organization. 27 Aug 2010; 85; 337-348. Available from: https://www.who.int/wer/2010/wer8535/en/.

2 Government of Nepal, Ministry of Health and Population (NP). Annual report. Kathmandu (NP): Department of Health Services; 2017/18.161p. Report No: 24.

3 Shrestha R, Shrestha S, Khanal P, KC B, Nepal's first case of COVID-19 and public health response. J Travel Medi, 2020; 27(3): 1-2. https://doi.org/10.1093/jtm/taaa02.12

4 ILEP Federation | Working worldwide to end leprosy. Available from: https://ilepfederation.org/. 